\title{
Diseño de Aplicación en Base a Modelo de Evaluación del Proceso de Enseñanza Aprendizaje en El Taller
}

\author{
A Design Studio Course Assessment Model Applied
}

\author{
Paola de la Sotta Lazzerini \\ FAU, Universidad de Chile \\ psotta@uchile.cl \\ Eduardo Hamuy Pinto \\ FAU, Universidad de Chile \\ ehamuy@uchile.cl
}

Bruno Perelli Soto

FAU, Universidad de Chile

bperelli@uchile.cl

\begin{abstract}
In design studio courses, assessment helps to improve teaching practice and student learning. Output-Mapping model considers a practical working method, applicable to teaching, based on records of students' outcomes. Visualization is a key component; iconographic resources aid understanding and observation of the territory conquered by each participant throughout the course. The next phase of the project addresses the need to manage large amounts of information generated by a statistical model for assessment. Visualization media used so far have proven limited. A new web system may dynamically visualize relationships between students' records more immediately, precisely and in a network.
\end{abstract}

Keywords: Design teaching; Assessment; Visualization.

\section{Introducción}

El diseño como disciplina considera en su ejercicio, y particularmente en la formación de diseñadores, una serie de metodologías que permiten conducir el proceso proyectual hacia una mirada holística del problema observado. La didáctica del Taller es flexible y responde a ese caudal humano (Mabardi 2012) con el que se cuenta para llevar a cabo los desafíos planteados durante su travesía. Se plantea un cuerpo organizado de actividades a resolver; donde se estipulan de manera jerárquica los temas a abordar según su nivel de complejidad cognitiva, como así también los criterios de desempeño específico requeridos para el correcto cumplimiento de los objetivos del taller. El problema surge entonces al momento de evaluar estos procesos, que incorporan aspectos de la percepción y apreciativos que interfieren muchas veces en valoraciones más objetivas.

La evaluación en las asignaturas teórico-prácticas, se enfoca principalmente hacia los logros adquiridos por el estudiante a través del desarrollo de proyectos. A pesar de su naturaleza predominantemente heurística y de las valoraciones cualitativas que muchas veces requieren las propuestas proyectuales, las evaluaciones formativas y sumativas por lo general se reducen a una valoración de carácter cuantitativo. Los instrumentos de evaluación tradicionales resultan deficientes o insuficientes como medio para advertir el proceso de evolución de un estudiante de modo más particular, integral y que potencie aquellas competencias que el estudiante trae. Se advierte entonces, que dentro del taller, se aplican instrumentos de evaluación no tradicionales, desarrollados por los docentes, con el fin de consignar aquellos aspectos que una escala de valoración cuantitativa no considera.

De igual forma el cambio en los paradigmas educativos, nos obliga a revisar la forma en que por un lado se enseña y se aprende (práctica docente) y por otro, lo que el mercado requiere del perfil profesional. La formación por competencias se incorpora al área de la educación, contribuyendo, pero no solucionando, la tarea del fortalecimiento en el proceso de enseñanza-aprendizaje; reconociendo que el ser humano se mueve en tres ejes virtuosos o dominios de competencia (cognitivo, procedimental y actitudinal) para la adquisición del conocimiento $y$ en los cuales puede apoyarse para fortalecer su propio proceso.

Se hace hincapié entonces, en el hecho de considerar la instancia de la evaluación como un componente, que busca no solo medir el nivel de logro alcanzado, sino también contribuye a la generación del ejercicio de la autoevaluación y co-evaluación de todo el grupo humano que participa en el proceso proyectual. La apropiación de una cultura de evaluación favorece a la capacidad de autonomía del estudiante para desarrollar y orientar su propio aprendizaje, como una destreza meta-cognitiva. 


\section{Metodología}

Con anterioridad se ha desarrollado el modelo Output Mapping (OM) que considera una metodología de trabajo práctico, aplicable a la didáctica del taller (De la Sotta, 2004; 2006; 2012). En el caso del modelo, la estrategia de enseñanza aprendizaje se contempla en unidades temáticas que se desarrollan a lo largo del año y que van de menor a mayor grado de complejidad. Para lo cual se considera un valor porcentual sobre cada una de ellas, acorde al nivel de competencia establecido por el programa. Como parte de la estrategia pedagógica, las unidades deben retroalimentarse entre ellas a medida que avanza el curso. De igual forma la didáctica a implementar en las unidades respectivas se enmarca por un lado en la aplicación de metodologías proyectuales, las que facilitan y contribuyen al reconocimiento de los pasos a tener en cuenta al momento de proyectar. Por otro lado, didácticas de trabajo grupal, experimentación en terreno, investigación aplicada, transversalidad con otras asignaturas y niveles, etc. En general las didácticas que se consideran guardan directa relación con el objetivo de aprendizaje que se desea alcanzar.

Tomando en consideración lo anterior se desarrolla un instrumento de evaluación integral que permita visualizar el proceso de enseñanza- aprendizaje del estudiante durante su tránsito por el taller en la carrera de diseño. Tomando para ello las áreas de competencia establecidas en la nueva reforma educacional que son: competencia conceptual (saber conocimientos) procedimental (saber hacer) y actitudinal (ser).

La estrategia de OM plantea el registro evolutivo, con observaciones, de cada sesión en una bitácora, que además consigna una breve biografía del estudiante. En el mapa de evaluación, el proceso de enseñanza aprendizaje se registra mediante la herramienta de la cartilla de evaluación con objetivos específicos según unidad. La cual permite corregir en forma continua, las posibles desviaciones que se generen en el camino, tanto por el estudiante como agente participativo de su proceso de enseñanza aprendizaje, como para el docente y su equipo. Se elabora entonces, una cartilla (Fig. 1) de evaluación (tabla de doble entrada), según la unidad a trabajar y sus ejercicios, que considera por un lado los parámetros de conocimiento que deben ser adquiridos acorde al nivel del programa académico, y por otro, los criterios de desempeño específico evaluables para cada uno de los ejercicios que se estimen necesarios para dar respuesta a esos parámetros. Una vez creada la cartilla, se procede a evaluar el resultado obtenido por el estudiante, mediante el cotejo con una rúbrica metafórica que permite observar el nivel de logro adquirido en ese criterio. De igual forma y una vez finalizado la mitad del periodo académico (semestre) se traspasa la información reflejada en las cartillas al mapa de evaluación donde el estudiante puede observar gráficamente su nivel de comportamiento y adquisición de logros según el dominio de aprendizaje.

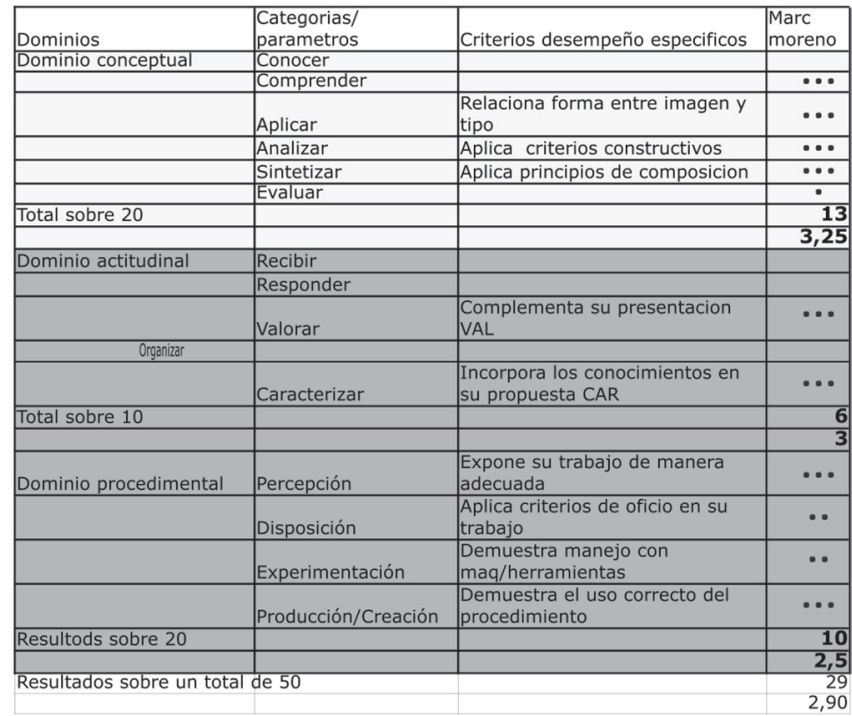

Figura 1: Cartilla de registro de las evaluaciones.

\section{Resultados}

Esta estrategia de evaluación ha sido validada en años recientes a través de sucesivos avances que han comprobado su validez como recurso semántico y cognitivo para docentes y estudiantes (De la Sotta, 2004; 2006; 2012). A través de prototipos experimentales que se utilizaron en las fases anteriores, se probaron empíricamente interfaces que representan el proceso de los estudiantes de taller a través de gráficos de líneas, barras, líneas de tendencias (Fig. 2) y gráficos radiales.

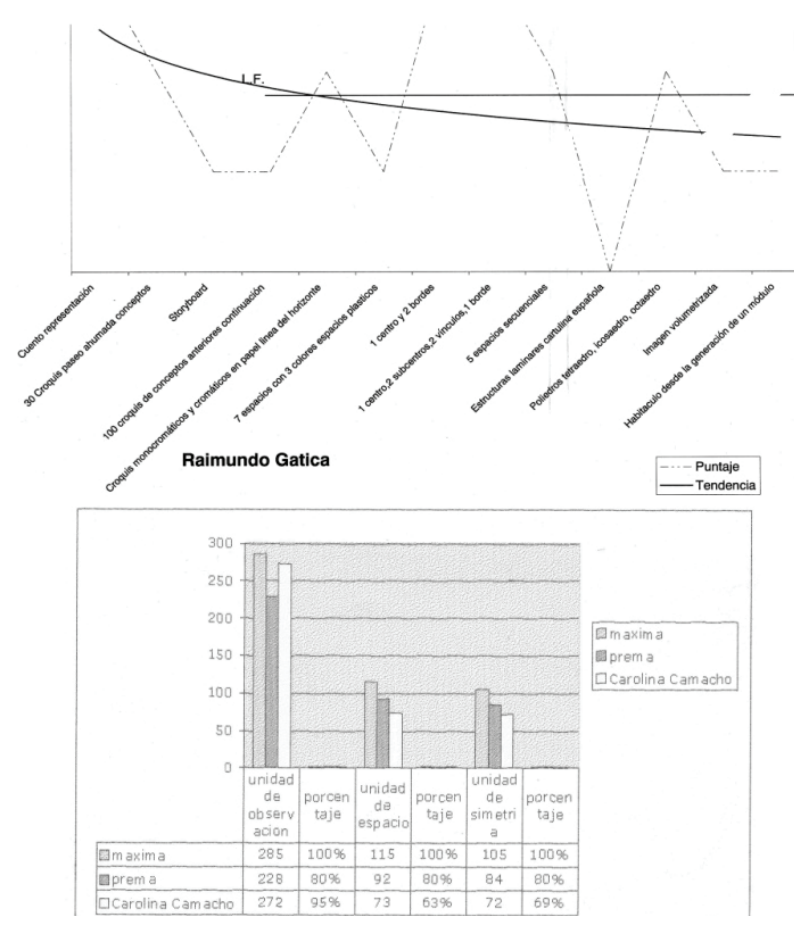

Figura 2: Primeras formas de visualización de OM. 
Las cartillas levantadas son representadas en OM, un gráfico radial (Fig. 3) elaborado con software de ofimática, que permite comprender y observar el logro alcanzado y el territorio de enseñanza aprendizaje a conquistar por cada participante, durante su tránsito por el Taller, al modo de un mapa. OM es entregado al estudiante en dos instancias durante el taller, a la mitad del programa académico y al final del mismo. El objetivo de lo anterior radica en que para que la visualización, como artefacto externo que apoya la toma de decisiones (Ware, 2013) sea efectivo, requiere de una serie de datos o conceptos para que puedan transformarse en información interpretable, si es bien presentada.

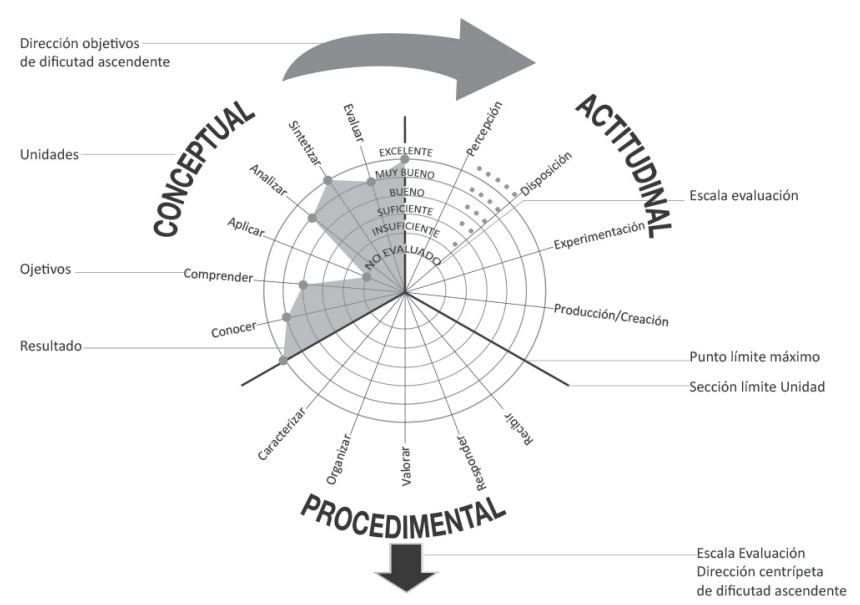

Figura 3: Estructura sintáctica y semántica de visualización de $\mathrm{OM}$ en gráfico radial, realizada con recursos de ofimática.

Se estipulan de manera jerárquica los temas a abordar según su nivel de complejidad cognitiva, como así también los criterios de desempeño específico requeridos para el mejor cumplimiento de los objetivos curriculares del taller. Los resultados de las sucesivas evaluaciones se exponen luego en una dinámica grupal de discusión abierta. De esta forma, OM se plantea como la representación de un cuerpo organizado de actividades a resolver dentro de una unidad académica y tiempo determinado, que considera la incorporación de la evaluación como parte del proceso formativo.

Como proyección de la investigación, recientemente se han realizado nuevos prototipos de visualización de OM (Fig. 4) con la finalidad de hacer más inmediata y dinámica la entrega de la representación del territorio conquistado por el estudiante a partir de su desempeño. Este artefacto de visualización, cuenta con un formulario online basado en la cartilla descrita con anterioridad. Este formulario, no sólo permite al equipo ingresar los valores basados en los logros del estudiante, sino que además es registrado a través de una base de datos que permite mantener un seguimiento en el tiempo del desempeño logrado proyecto a proyecto.

\section{Conclusiones}

OM permite al equipo docente, ajustar la didáctica aplicada y advertir posibles errores en cuanto a comprensión de contenidos, y explicación de procedimientos, entre otras.

Todo ello contribuye a la consideración de la evaluación como una herramienta de aplicación constante, promoviendo al fortalecimiento de la autoevaluación, coevaluación y metaevaluación para todos los actores involucrados, incluyendo la institución.

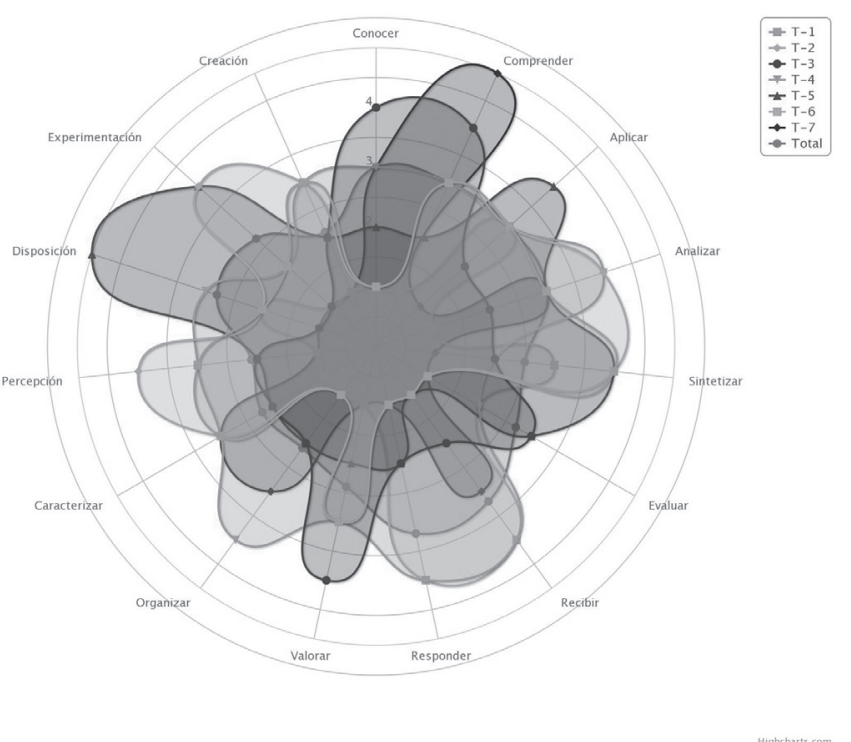

Figura 4: Prototipo de visualización realizada con formulario dinámico.

El recurso iconográfico permite comprender y observar el logro alcanzado y el territorio de enseñanza aprendizaje a conquistar por cada participante, durante su tránsito por el Taller. La representación gráfica o visualización del proceso de enseñanza aprendizaje de un estudiante, apela mediante un lenguaje familiar, a una lectura más analítica sobre el territorio conquistado. Toda vez que la síntesis formal y el color son instrumentos propios de la disciplina proyectual. Se observa la necesidad de desarrollar mejor su forma de aplicación a través del diseño de una herramienta computacional que pueda ser utilizada por equipos docentes en talleres de diseño.

La Visualización es un campo del conocimiento en sí mismo, un campo interdisciplinario de investigación (Chen, 2006). Con diferentes acepciones en la literatura, coinciden en hacer referencia a una representación mental de carácter visual y a su cualidad como herramienta cognitiva (Mazza, 2009; Ware, 2012). En su acepción más tradicional, ella se entiende como una representación mental de naturaleza visual, o como una representación visual externa facilitadora de la actividad cognitiva (Mazza, 2009). La visualización, será considerada como el proceso cognitivo resultante de la interacción con el artefacto de visualización (Mazza, 2009). La construcción de esta imagen 
mental, se apoya en procesos de percepción, principios de la Gestalt, y estimulación visual (Ware, 2008).

Se pueden establecer dos instancias de visualización, una visualización de índole mental o cognitiva, donde efectivamente se establece una imagen mental de un concepto abstracto, y por otra parte, tenemos una visualización de índole funcional, en la cual interactuamos con datos cualitativos o cuantitativos, de carácter estadístico, que son procesados a través de un artefacto visual destinado a representar mediante imágenes de fenómenos de otro carácter.

A partir de esta distinción es que definiremos como visualización de datos a una extensa colección de técnicas gráficas para el proceso y representación de datos, que puede dirigir la investigación y análisis de cualquier conjunto de datos multivariante. Existen varias técnicas que pueden ser utilizadas para describir relaciones dentro de datos mientras la inteligencia busca el camino más adecuado para que un espacio multidimensional sea proyectado en dos dimensiones. Estas técnicas pueden ser utilizadas en actividades como descubrimiento de conocimiento, predicción, modelamiento, desarrollo de tareas inteligentes, reconocimiento de patrones, etc. (Kosti, 2011). Dzemyda, Kurasova y Žilinskas (2013) dan el nombre de Visualización Directa a una presentación gráfica de un conjunto de datos que provee una comprensión cualitativa de la información en una forma natural y directa.

Para el futuro trabajo, nosotros utilizamos el término Visualización al igual que Ware (2012) para referirnos a un artefacto que apoya la toma de decisiones a través de la representación gráfica de datos y/o conceptos.

En la investigación de la visualización de información se destacan cuestiones fundamentales. Resulta un desafío para los diseñadores identificar las estrategias y herramientas más apropiadas para visualizar un determinado tipo de información. La visualización facilita el análisis y la posterior comprensión de los fenómenos y comportamiento emergente presentes en los sistemas complejos y conjuntos de datos dimensionales más altos. Desarrollos relativamente recientes en el hardware de visualización han hecho posible la manipulación de conjuntos de datos grandes y complejos en tiempo real para llevar a cabo la recuperación de información, minería de datos y el procesamiento analítico en línea (Liere, Adriaansen, \& ZudilovaSeinstra, 2009). La visualización implica un gran número de estructuras de representación y el diseño de sistemas que integren la gestión intensiva de información (Chen, 2006). Además, en el campo de la visualización, surgen regularmente nuevas formas de representación e interacción con ella (Dzemyda et al., 2013; Liere et al., 2009; Ware, 2012). Resulta mucho más recurrente el estudio de la visualización de datos cuantitativos (Chen, 2006; Mazza, 2009; Ware, 2012) que el de información cualitativa, como por ejemplo caracteriza la evaluación en el aprendizaje del proceso proyectual (Cash, Stanković, \& Štorga, 2013). Por tanto este es un desafío adicional.
Siendo la visualización un componente fundamental de OM, se hace necesario responder. ¿Cuál recurso de visualización resulta más adecuado para implementar en la aplicación de OM?

Responder esta pregunta es el objetivo que nos hemos fijado para las siguientes fases de investigación de la aplicación del modelo OM.

\section{Referencias}

Cash, P., Stanković, T., \& Štorga, M. (2013). Using visual information analysis to explore complex patterns in the activity of designers. Design Studies, 1-28. doi:10.1016/j.destud.2013.06.001 (in press)

Chen, C. (2006). Information Visualization: Beyond the Horizon (2nd ed., p. 332). Springer. doi:10.1007/SpringerReference_6313

De la Sotta, P. (2004) Informe Final: Un modelo de evaluación para la visualización del proceso de enseñanza - aprendizaje en el estudiante de diseño. Concurso de Proyectos de Investigación y Experiencias Docentes, MECESUP UCH 0217 (No publicado).

De la Sotta, P. (2006) Informe Final: Un modelo de evaluación para la visualización del proceso de enseñanza - aprendizaje en el estudiante de diseño. II parte 2005-2006. Concurso de Proyectos de Investigación y Experiencias Docentes, MECESUP UCH 0217 (No publicado).

De la Sotta, P. (2012) Output Mapping. Documento presentación defensa para optar a Magíster en Didáctica Proyectual, Universidad del Bíobío, Concepción, noviembre 2012. (Comunicación personal).

De la Sotta, P. (2013) Output Mapping: Modelo de evaluación del proceso de enseñanza aprendizaje en la asignatura de Taller. IV Congreso de Enseñanza del Diseño Universidad de Palermo. 29-31 julio, Buenos Aires: Argentina

Dzemyda, G., Kurasova, O., \& Žilinskas, J. (2013). Multidimensional Data Visualization (Vol. 75). New York, NY: Springer New York. doi:10.1007/978-1-4419-0236-8

Huang, W. (Ed.). (2014). Handbook of Human Centric Visualization. New York, NY: Springer New York. doi:10.1007/978-1-4614-7485-2

Hundhausen, C. D. (2014). Evaluating Visualization Environments: Cognitive, Social, and Cultural Perspectives. In W. Huang (Ed.), Handbook of Human Centric Visualization (pp. 115-145). New York, NY: Springer New York. doi:10.1007/978-1-4614-7485-2

Liere, R., Adriaansen, T., \& Zudilova-Seinstra, E. (Eds.). (2009). Trends in Interactive Visualization. London: Springer London. doi:10.1007/9781-84800-269-2

Mabardi, J.-F. (2012). Maestría del proyecto. Apuntes para la práctica de la enseñanza del proyecto. (R. Lagos, Ed.) (1st ed., p. 191). Concepción: Chile: Ediciones Universidad del Bío-Bío.

Mazza, R. (2009). Introduction to Information Visualization (p. 139). London: Springer London. doi:10.1007/978-1-84800-219-7

Rugiero, A., Apip, A., \& Hirmas, A. (2006). Evaluación del aprendizaje autoconstruido. Santiago de Chile: Facultad de Arquitectura y Urbanismo de la Universidad de Chile.

Universidad de Chile. (2010). Modelo Educativo. Santiago: Chile. (p. 20).

Ware, C. (2008). Visual Thinking: For Design. (S. Card, J. Grudin, \& J. Nielsen, Eds.) Ergonomics (Vol. 53). Morgan Kaufmann.

Ware, C. (2012). Information Visualization: Perception for Design (Interactive Technologies) (3rd ed.). Morgan Kaufmann. 